\title{
MIDLIFE CIGARETTE SMOKING AND NEUROPSYCHIATRIC SYMPTOMS AMONG DEMENTED OUTPATIENTS
}

\author{
A. McMurtray ${ }^{1,2,3}$, V. Krishna ${ }^{4}$ B. Nakamoto ${ }^{5,6}$, N. Diaz ${ }^{1,2,3}$, B. Mehta ${ }^{1,2,3}$, S. Aboutalib ${ }^{2}$, E. Saito ${ }^{1}$
}

\begin{abstract}
Background: Midlife cigarette smoking is associated with increased risk for both midlife neuropsychiatric symptoms and development of dementia later in life. Objective: This study was designed to extend knowledge of these relationships by assessing for increased risk of neuropsychiatric symptoms among demented outpatients related to midlife smoking habits. Design: A retrospective cross-sectional analysis. Setting: Patients seen in a community based outpatient clinic for treatment of dementia during a one year period. Participants: A total of 38 participants were included in this study, 22 with a history of midlife smoking and 16 lifetime non-smokers. Results: Midlife cigarette smoking was associated with midlife alcohol use $(p=0.023)$ and presence of delusions ( $\mathrm{p}=0.031)$ among demented outpatients. Conclusions: A history of midlife smoking is associated with increased frequency of delusions later in life among demented outpatients and may help identify those at higher risk for developing neuropsychiatric symptoms.
\end{abstract}

Key words: Dementia, Alzheimer's disease, smoking, delusions.

\section{Introduction}

High rates of cigarette smoking have previously been reported among individuals with psychiatric disorders such as depression, anxiety, and psychosis (1). Previous estimates suggest that individuals with psychiatric disorders account for up to $46 \%$ of all cigarette use in the United States (2), and that smoking prevalence among individuals with psychiatric disorders may be as high as $41 \%$, compared to only $22 \%$ among individuals without psychiatric disorders (3).

The high co-morbidity between cigarette smoking and psychiatric disorders has been suggested to occur due to significant alterations in functioning of brain cholinergic and other neurotransmitter systems $(1,4,5)$. Nicotine is reported to affect several neurotransmitter systems involved in development of psychiatric disorders. The dopamine system is the most well studied neurotransmitter system affected by nicotine intake from

1. Neurology Division, Los Angeles Biomedical Research Institute, Torrance, CA USA; 2. Neurology Department, Harbor-UCLA, Torrance, CA, USA; 3. Neurology Department, David Geffen School of Medicine, University of California at Los Angeles, Los Angeles, CA, USA; 4. Boston College, Chestnut Hill, MA; 5. Neurology Department, John A. Burns School of Medicine, University of Hawaii, Honolulu, HI, USA; 6. Neurology Department, Straub Hospital and Clinics, Honolulu, HI, USA.

Corresponding Author: Aaron McMurtray, M.D., Ph.D., Neurology Department Building N-25, Harbor-UCLA Medical Center, 1000 West Carson Street, Torrance, CA 90509. USA. Phone (310) 222-3897. Fax: (310) 533-8905. E-mail: amcmurtray@dhs.lacounty.gov. cigarette smoking, with brain dopamine levels altered through activation of nicotinic acetylcholine receptors on mesolimbic interneurons (6-8). Other neurotransmitter systems altered by activation of nicotinic cholinergic neurons due to smoking are less well studied and include: endogenous opioid peptides, gamaaminobutyric acid, glutamate, norepinephrine, and serotonin systems (8). Smoking induced modulation of these neurotransmitter systems links cigarette smoking to neurotransmitter imbalances associated with psychiatric disorders. For this reason, cigarette smoking has even been described as a possible form of attempted selfmedication as these patients try to correct dysfunction of cholinergic and other neurotransmitter systems that may be associated with their psychiatric symptoms $(1,4)$.

This study was designed to extend knowledge of these relationships by assessing for increased risk of neuropsychiatric symptoms among demented outpatients related to midlife smoking habits. We hypothesized that there is an increased prevalence of neuropsychiatric symptoms such as depression, anxiety, delusions, and hallucinations among dementia patients who have a history of cigarette smoking compared to those who did not smoke. We were particularly interested in patients with Alzheimer's Disease as the etiology of their dementing illness since this condition is associated with a gradually developing alteration of brain cholinergic function that may precede development of cognitive symptoms by many years or even start during 
midlife.

\section{Methods}

Subjects: Participants were adults, over the age of 18 years, who presented sequentially to a community-based dementia subspecialty clinic during a one-year period from January 1st 2012 to January 1st 2013 for evaluation and treatment of dementia. De-identified data was obtained and analyzed in a retrospective fashion for all participants. Local Institutional Review Board approval was obtained.

Standardized Evaluation: All patients underwent a standardized workup consisting of a detailed history, general physical and neurological examinations, a brain imaging study with either computed tomography or magnetic resonance imaging, laboratory blood tests for treatable causes of cognitive impairment including serum vitamin B12, folate, and TSH levels, as well as RPR and HIV testing, and a Mini-Mental State Examination (9). Midlife smoking status was determined by either selfreport or report by family members or caregivers if selfreport was considered unobtainable or unreliable by the examining neurologist.

Dementia Diagnosis: Diagnoses of dementia were made by a board certified neurologist according to established clinical criteria (10). Dementia severity was determined by MMSE score with mild dementia indicated by scores ranging from 21 to 25 , moderate dementia by scores ranging from 10 to 20 , and severe dementia by scores below 10. Presence of neuropsychiatric symptoms including depression, anxiety, delusions and hallucinations were determined by a board certified neurologist during the history and physical examination.

Statistics: Normally distributed continuous demographic factors and other continuous variables were compared between groups using two-tailed t-tests. Continuous non-parametric data was compared between groups using the Mann-Whitney U test. Frequency of occurrence of categorical variables was compared between groups using chi-square analysis or Fisher's exact test as appropriate. All statistical calculations were performed using IBM SPSS Statistics for Windows, version 21.0.

\section{Results}

A total of 79 patients presented to the community based dementia subspecialty clinic during the one-year retrospective study period. Of these, 38 patients met clinical diagnostic criteria for either possible or probable Alzheimer's disease and were included in this retrospective analysis. Of the study participants, 22 had a history of midlife smoking and 16 were lifetime nonsmokers. A total of 28 patients were excluded because they did not meet clinical criteria for either dementia and a total of 13 patients were excluded because an adequate and reliable midlife smoking history could not be obtained.

Overall midlife smoker and lifetime non-smoker groups were very similar and did not significantly differ in demographic factors such as mean age, dementia onset age, gender distribution, ethnicity distributions, or presence of other significant co-morbid medical problems such as hypertension, diabetes, coronary artery disease or previous stroke (See Table 1). There was a trend towards lower mean MMSE scores among the lifelong non-

Table 1

Demographic Factors of Midlife Smokers and Non-Smokers

\begin{tabular}{|c|c|c|c|}
\hline $\begin{array}{l}\text { Demographic Factors and } \\
\text { Neuropsychiatric Symptoms }\end{array}$ & $\begin{array}{l}\text { Midlife Smokers } \\
\qquad \mathrm{N}=22\end{array}$ & $\begin{array}{l}\text { Non-Smokers } \\
\qquad \mathbf{N}=16\end{array}$ & Sig. \\
\hline Mean age (S.D.) ${ }^{\mathrm{a}}$ & $66.42(10.705)$ & $68.31(11.294)$ & $\mathrm{p}=0.693$ \\
\hline Mean age of onset (S.D.) & $61.74(9.036)$ & $60.31(10.912)$ & $\mathrm{p}=0.337$ \\
\hline Mean MMSE score (S.D.) & $17.76(6.796)$ & $13.86(9.670)$ & $\mathrm{p}=0.074$ \\
\hline Male & $8(36.4 \%)$ & $9(56.3 \%)$ & $\mathrm{p}=0.478$ \\
\hline Ethnicity (AA, A, C, H, Other/ unidentified) ${ }^{\mathrm{b}}$ & $\begin{array}{c}3(13.6 \%), 3(13.6 \%), 13(59.1 \%) \\
3(13.6 \%), 0(0.0 \%)\end{array}$ & $\begin{array}{c}3(18.8 \%), 2(11.7 \%), 9(56.3 \%), \\
1(6.3 \%), 1(6.3 \%)\end{array}$ & $\mathrm{p}=0.967$ \\
\hline Hypertension & $12(54.5 \%)$ & $9(56.3 \%)$ & $\mathrm{p}=0.974$ \\
\hline Diabetes & $5(22.7 \%)$ & $4(25.0 \%)$ & $\mathrm{p}=0.277$ \\
\hline Coronary Artery Disease & $2(9.1 \%)$ & $0(0.0 \%)$ & $\mathrm{p}=0.565$ \\
\hline Atrial Fibrillation & $1(4.5 \%)$ & $1(6.3 \%)$ & $\mathrm{p}=0.309$ \\
\hline Congestive Heart Failure & $2(9.1 \%)$ & $0(0 \%)$ & $\mathrm{p}=0.565$ \\
\hline Stroke & $4(18.2 \%)$ & $3(18.8 \%)$ & $\mathrm{p}=0.718$ \\
\hline Dyslipidemia & $6(27.3 \%)$ & $7(43.8 \%)$ & $\mathrm{p}=0.331$ \\
\hline Alcohol Use & $6(27.3 \%)$ & $0(0.0 \%)$ & $\mathrm{p}=0.023$ \\
\hline Illicit Substance Use & $3(13.6 \%)$ & $4(25.0 \%)$ & $\mathrm{p}=0.372$ \\
\hline
\end{tabular}

†S.D. = Standard Deviation; $¥ \mathrm{AA}=$ African American, $\mathrm{A}=$ Asian, $\mathrm{C}=$ Caucasian, $\mathrm{H}=$ Hispanic. 
Table 2

Neuropsychiatric Symptoms among Midlife Smokers and Non-Smokers

\begin{tabular}{|c|c|c|c|}
\hline Neuropsychiatric Symptoms & Midlife Smokers $(\mathrm{N}=22)$ & Non-Smokers $(\mathrm{N}=16)$ & Sig. \\
\hline Anxiety & $7(31.8 \%)$ & $3(18.8 \%)$ & 0.366 \\
\hline Delusions & $10(45.5 \%)$ & $2(12.5 \%)$ & 0.031 \\
\hline Depression & $13(59.1 \%)$ & $9(56.3 \%)$ & 0.646 \\
\hline Hallucinations & $7(31.8 \%)$ & $5(31.3 \%)$ & 0.743 \\
\hline Paranoia & $7(31.8 \%)$ & $1(6.3 \%)$ & 0.056 \\
\hline Disruptive Behaviors & $8(36.4 \%)$ & $9(56.3 \%)$ & 0.224 \\
\hline Wandering & $8(36.4 \%)$ & $2(12.5 \%)$ & 0.099 \\
\hline
\end{tabular}

smokers $($ mean $=13.86$, S.D. $=9.670, \mathrm{p}=0.074)$ compared to midlife smokers $($ mean $=17.76$, S.D. $=6.796)$. While midlife cigarette smoking was positively associated with midlife alcohol use $(p=0.023)$, no significant relationship was detected with midlife illicit substance use $(p=0.372)$.

Neuropsychiatric symptoms were present in 22 of the 38 patients $(57.89 \%)$, including 13 of the 22 midlife smokers $(59.09 \%)$ and 9 of the 16 lifelong non-smokers $(56.25 \%)$. Overall for the entire group, anxiety was present in $26.32 \%$ of the participants, delusions in $31.58 \%$, depression in $57.89 \%$, hallucinations in $31.58 \%$, paranoia in $21.05 \%$, disruptive behaviors in $44.74 \%$, and wandering in $26.32 \%$ of the patients (See Table 2). There was no significant relationship between midlife cigarette smoking and presence of anxiety $(\mathrm{p}=0.399)$, depression $(p=0.646)$, hallucinations $(p=0.743)$, or disruptive behaviors $(p=0.224)$. Midlife cigarette smoking, however, was positively associated with presence of delusions $(p=0.031)$. Additionally, trends were noted towards greater frequency of paranoia $(p=0.056)$ and wandering behaviors $(\mathrm{p}=0.099)$ among midlife smokers compared to non-smokers (See Table 2).

\section{Discussion}

In this study we identified an association between midlife cigarette smoking and increased frequency of delusions later in life among a community-based sample of demented outpatients. Because cortical cholinergic deficits are known to occur in both Alzheimer's disease and vascular dementia, it is possible that midlife cigarette smoking may represent a form of self-medication used by these patients to treat subtle cholinergic disturbance present years or even decades before frank dementia is evident. The findings of this study suggest that a history of midlife cigarette smoking may help identify dementia patients at increased risk for development of neuropsychiatric symptoms.

Presence of neuropsychiatric symptoms among dementia patients is associated with greater cost of providing care at all stages of dementia severity (11). The greater care costs occur primarily due to increased time needed for providing direct help and supervision to these patients. One recent study quantified the average increase in direct help and supervision related to the number of neuropsychiatric symptoms present, reporting an additional 10.0 hours of active help and 12.4 hours of supervision required per week for those with 1-2 neuropsychiatric symptoms. Those with 3 or more neuropsychiatric symptoms required 18.2 hours of active help and 28.7 hours of supervision per week (11). Consequently, determining factors that may predict development and identify risk of neuropsychiatric symptoms among dementia patients could allow for earlier intervention and possibly reduce care costs.

In this study, 22 of the 38 demented individuals included $(58 \%)$ reported a positive midlife smoking history. This is somewhat greater than the percentage described in previous reports which typically range between $46-48 \%(12,13)$. Similarly, the prevalence of neuropsychiatric symptoms identified in this study was lower than previous reports. Recent prevalence reports for rates of at least one neuropsychiatric symptom among demented individuals range from $69-82 \%(14,15)$, which is greater than the prevalence rate of $58 \%$ described in this study. The greater prevalence of neuropsychiatric symptoms identified in the prior studies is likely due to differences in the populations being studied, with our study including relatively fewer nursing home residents and more community dwelling individuals.

This study has several limitations. First, the cohort studied consisted of a small convenience sample that was heavily over-represented by Caucasian participants. Consequently the generalizability of the results to ethnicities not better represented in the study would require further investigation. The small sample size may have also contributed to the lack of associations detected between midlife cigarette smoking and other neuropsychiatric symptoms, and it is possible that a similar assessment of a larger database may yield different results. This study was also hampered by the lack of a standardized tool for assessment of neuropsychiatric symptoms as well as for assessment and quantification of midlife smoking habits. Additionally, the diagnosis of dementia was made by a single 
investigator and was based on clinical examination only, consequently the inter-rater reliability of the diagnosis could not be determined and there is no pathological confirmation of the clinical diagnosis.

As rates of dementia are expected to continue to rise in coming years, understanding and predicting occurrence of neuropsychiatric symptoms in this population is likely to be a topic of increasing importance. The association identified in this study between midlife smoking habits and presence of delusions suggests that midlife smoking history may help to identify dementia patients at increased risk for future development of neuropsychiatric symptoms. Further study is needed to confirm and better understand the association described in this report. Particularly useful would be a study of a larger, well characterized demented population that includes quantitative assessment of midlife smoking habits such as pack-year smoking histories and data obtained from use of standardized assessment tools for detection of neuropsychiatric symptoms.

Funding: This work was supported by CTSI Grant UL1TR000124.

Conflicts of interest: None of the authors report any conflicts of interests.

\section{References}

1. D'Souza MS, Markou A. Schizophrenia and tobacco smoking comorbidity: nAChR agonists in the treatment of schizophrenia-associated cognitive deficits. Neuropharmacology 2012;62:1564-1573.
2. Grant BF, Hasin DS, Chou SP, Stinson FS, Dawson DA. Nicotine dependence and psychiatric disorders in the United States: results from the National Epidemiologic Survey on Alcohol and Related Conditions. Arch Gen Psychiatry 2004;61:1107-1115.

3. Lasser K, Boyd JW, Woolhandler S, Himmelstein DU, McCormick D, Bor DH. Smoking and Mental Illness: a population-based study. JAMA 2000;284:26062610.

4. Kalman D, Morissette SB, George TP. Co-Morbidity of Smoking in Patients with Psychiatric and Substance Use Disorders. AM J Addict 2005;14:106-123.

5. Mineur YS, Picciotto MR. Nicotine receptors and depression: revisiting and revising the cholinergic hypothesis. Trends Pharmacol Sci 2010;31:580-86.

6. Moran LV, Sampath H, Kochunov P, Hong LE. Brain circuits that link schizophrenia to high risk of cigarette smoking. Schizophr Bull 2013;39:13731381.

7. George TP, O'Malley SS. Current pharmacological treatments for nicotine dependence. Trends Pharmacol Sci 2004;25:42-48.

8. Picciotto MR. Nicotine as a modulator of behavior: beyond the inverted U. Trends Pharmacol Sci 2003;23:494-499.

9. Folstein MF, Folstein SE, McHugh PR. Mini-mental state. A practical method for grading the cognitive state of patients for the clinician. J Psychiatr Res 1975;12:189-198.

10. Sorbi S, Hort J, Erkinjuntti T, et al. EFNS-ENS Guidelines on the diagnosis and management of disorders associated with dementia. Eur J Neurol 2012;19:1159-1179.

11. Okura T, Langa KM. Caregiver Burden and Neuropsychiatric Symptoms in Older Adults with Cognitive Impairment: The Aging, Demographics, and Memory Study (ADAMS). Alzheimer Dis Assoc Disord 2011;25:116-121.

12. Kivipelto M, Helkala EL, Laakso MP, et al. Midlife vascular risk factors and Alzheimer's disease in later life: longitudinal, population based study. BMJ 2001;322:1447-1451.

13. Van Duijn CM, Hofman A. Relation between nicotine intake and Alzheimer's disease. BMJ 1991;302:1491-1494.

14. Selbaek G, Engedal K, Bergh S. The prevalence and course of neuropsychiatric symptoms in nursing home patients with dementia: a systematic review. J Am Med Dir Assoc 2013;14:161-169.

15. D'Onofrio G, Sancarlo D, Panza F, et al. Neuropsychiatric symptoms and functional status in Alzheimer's disease and vascular dementia patients. Curr Alzheimer Res 2012;9:759-771. 